\title{
Lenguaje y dispositivo. Un análisis de la serie Dr. House como caso paradigmático de la práctica médica colombiana
}

\section{Jairo Clavijo Poveda}

PhD en Paris III Antropólogo de la Universidad de Los Andes, Bogotá-Colombia. Profesor titular de la Pontificia Universidad Javeriana, tutor del semillero de estudios estructuralistas de la Javeriana y miembro del grupo Bioetica.

jairo.clavijo@javeriana.edu.co

\section{Juan Camilo Ospina Deaza}

Antropólogo de la Pontificia Universidad Javeriana de Bogotá-Colombia, profesor de catedra, coordinador del semillero de estudios estructuralistas y miembro del grupo Bioetica de la Javeriana.

jospinad@javeriana.edu.co

\section{Valeria Sanchez Prieto}

Estudiante de antropología de la Pontificia Universidad Javeriana de Bogotá-Colombia. Coordinadora del semillero de investigacion de estudios estructuralistas de la Javeriana y guia en recepcion del Museo Arqueologico Casa del Marqués de San Jorge.

sanchez.valeria@javeriana.edu.co

\section{Resumen}

En el marco de la filosofía de la ciencia y la tecnología, se resalta la importancia de la conversión del cuerpo a un lenguaje médico, dentro de la practica médica, vista a través de la serie televisiva Doctor House. Se seleccionaron apartados de tres capítulos de la serie, a pesar de que los casos que se abordan en la serie son ficticios, las situaciones que aparecen son similares a situaciones reales y por tanto son susceptibles de ser interpretadas. Retomamos las contribuciones de Giorgio Agamben sobre el "dispositivo" para analizar la expansión del poder médico en la actualidad. El médico al ejercer su oficio hace una conversión de lo viviente a códigos, índices y conceptos. El proceso de diagnóstico es siempre un proceso lingüístico, basado en la observación por parte del médico de síntomas y signos de la enfermedad. Los médicos tienen unas prótesis físicas que les permiten percibir el cuerpo de los pacientes de forma diferente a la manera que lo harían sólo con los sentidos humanos, como resultado tienen la capacidad de convertir lo viviente en datos.

\section{Palabras clave}

Dispositivo, Datos, Estrategias del poder, Poder medico, Agamben. 


\title{
Language and dispositive. An analysis of the TV show Dr. House as a paradigmatic case of Colombian medical practice
}

\author{
Jairo Clavijo Poveda \\ PhD in Paris III Anthropologist of the University of Los Andes, Bogotá-Colombia. Full \\ professor of the Pontificia Universidad Javeriana, tutor of the seedbed of structuralist \\ studies of the Javeriana and member of the Bioetica group. \\ jairo.clavijo@javeriana.edu.co
}

\section{Juan Camilo Ospina Deaza}

Anthropologist of the Pontificia Universidad Javeriana de Bogotá-Colombia, professor professor, coordinator of the seedbed of structuralist studies and member of the Bioetica de la Javeriana group.

jospinad@javeriana.edu.co

\section{Valeria Sanchez Prieto}

Anthropology student of the Pontificia Universidad Javeriana de Bogotá-Colombia. Coordinator of the research hotbed of structural studies of the Javeriana and guide in reception of the Archaeological Museum Casa del Marqués de San Jorge.

sanchez.valeria@javeriana.edu.co

\begin{abstract}
In the framework of the Philosophy of Science and Technology, this article wants to highlight the importance of the conversion of the body into a medical language. In order to achieve this goal, we made use of the Television Show Dr. House. We selected different fragments of three episodes and, even though the cases shown by the program are not real they keep similarities with real life cases and, in this sense, are susceptible to be analyzed. We based our analysis in the agambenian notion of "dispositive" to understand the extension of medical power nowadays. A doctor, to exercise his trade, converts the living into codes, index and concepts. The diagnosis process is always a linguistic process, based on the observation by the doctor and the selection of symptoms and signs of sickness. Doctors have physical prosthesis that allow them to perceive the patient's body in a different way that if they only depended upon their human senses, consequently they are able to convert the body into data.
\end{abstract}

\section{Keywords}

Dispositive, Data, Power strategies, Medical power, Agamben. 


\section{Introducción}

En este artículo se resalta la importancia de la conversión del cuerpo a un lenguaje médico, dentro de la práctica médica, vista a través de la serie televisiva Doctor House. En primer lugar, exploramos qué es una tecnología, en el marco de la filosofía de la ciencia y la tecnología, en la práctica médica. En segundo lugar, exponemos qué es el lenguaje en la práctica médica, y tomamos el caso de Dr House como ejemplo. Para lograr lo anterior, damos un breve contexto de la serie televisiva, y abordamos fragmentos de algunos apartados de la serie, con el fin de poner en evidencia los elementos que componen el dispositivo médico. Se seleccionaron apartados de tres capítulos de la serie, tomándose, como objeto de análisis, situaciones concretas del capítulo 14 de la primera temporada, 10 de la segunda temporada, y 3 de la tercera temporada de la serie. Finalmente, será a partir del análisis que se comprenderá a la práctica médica como un dispositivo.

Para este punto, los lectores podrán preguntarse por qué hablar de una serie televisiva para comprender estas dinámicas de la medicina. A pesar de que los casos que se abordan en la serie son fictícios, las situaciones que aparecen son similares a situaciones reales, y por tanto son susceptibles de ser etnografiadas e interpretadas. Además, consideramos que la serie muestra un ideal de la práctica médica que puede apreciarse en la realidad de la atención médica. Este estudio se realizó bajo el marco conceptual de la perspectiva de la filosófia de la ciencia y la tecnología, comprendida desde la antropología. El objetivo de este artículo es poner en evidencia la existencia de un lenguaje médico que codifica el cuerpo humano, convirtiéndolo en datos.

\section{¿Qué es una tecnología? La filosofía de la ciencia y la tecnología en la práctica médica}

Los estudios sociales sobre ciencia y tecnología abarcan un campo transdisciplinar de estudios sobre los efectos culturales, éticos, y políticos del conocimiento científico y la innovación tecnológica. Colocan el énfasis en la interpretación sobre las utilidades, apropiaciones e impactos en la vida cotidiana de las personas, con el objetivo de romper las antiguas barreras de investigación científico-técnica. Sus actuales exponentes incluyen a figuras como: Bruno Latour, Michael Lynch, y Harold Garfinkel, entre otros. Esta perspectiva se originó en Europa y Estados Unidos. Estos problemas no son ajenos a la antropologia, como puede evidenciarse en el trabajo de Byron Good (1994), Didier Fassin (2004), Ana Cristina Pertierra (2018), entre muchos otros.

En los tiempos contemporáneos, la tecnología y la ciencia son una parte importante de la vida cotidiana. Preguntas como: ¿Qué papel juega la tecnología en la experiencia humana cotidiana?, ¿cómo afectan los artefactos tecnológicos la existencia de las personas y sus relaciones con el mundo?, y ¿cómo los instrumentos producen y transforman el conocimiento humano? (Verbeek, 2001: 119) son preguntas pertinentes para la antropología contemporánea. 
Por lo general, la tecnología se nos presenta como un objeto independiente de las relaciones humanas; se piensa generalmente como un objeto o dispositivo (device). Como resultado, Verbeek afirma que "la filosofía clásica de la tecnología tiende a reificar la tecnología, tratándola como una «fuerza monolítica»" (Verbeek, 2001: 120). Sin embargo, ¿qué significa que la tecnología sea "reificada"? Desde el punto de vista de Marx y Lukács, la reificación significa tratar las relaciones humanas como objetos debido a las relaciones de mercado. Por lo tanto, para comprender adecuadamente la tecnología, es necesario contrastar nuestra definición inicial.

La tecnología está atravesada por procesos de producción, circulación y consumo, en consecuencia es más que un mero objeto (device), es el producto de relaciones complejas. Es importante llevar mas allá esta interpretación, y darse cuenta que, como Heidegger afirmó, "la tecnología moderna debe entenderse no como la suma total de dispositivos tecnológicos, sino como las formas en que nuestra época actual revela el mundo", o, como Foucault lo entiende, "procedimientos que han sido inventados, perfeccionados y desarrollados sin cesar" (Foucault, 1994: 189).

Si una tecnología no es necesariamente un objeto, ¿podría ser algo más, una práctica, una forma de leer, un grupo de acciones, un objeto? Desde nuestra perspectiva, la tecnología abarca todos estos elementos mencionados. A veces, la tecnología también se entiende como una herramienta; Heidegger caracteriza la herramienta como "«something in order to»; it is serviceable, helpful, usable" (Heidegger,1996: 64). De la misma manera, Ihde encuentra que las herramientas están vinculadas a un contexto, "este campo dentro del cual una herramienta es lo que puede ser, es un espacio complejo lleno de «implicaciones» o «relaciones cruzadas»" (Ihde, 1990: 32). Señalar el contexto significa comprender para qué se usa, cómo se usa, qué se requiere para usar el objeto.

Por ejemplo, siguiendo a Canguilhem (2008), el microscopio es el resultado de la objetivación del conocimiento. Por lo tanto, un microscopio solo tiene sentido debido a los hallazgos de la biología, y la capacidad de un grupo de personas que entienden lo que muestra el objeto. Cuando Ihde menciona: "la tecnología no existe en sí misma, sino en relación con una cultura" (Ihde en Verbeek, 2001: 120), significa que la tecnología necesita de la cultura para que se pueda construir el objeto y, asimismo, la tecnología requiere los conocimientos culturales para ser usada. Es posible observar esta relación cuando hablamos de ciencia y tecnología, "los instrumentos median las percepciones de los científicos y los transforman en procesos. (...) Los instrumentos hacen que las cosas sean legibles a través de 'visualizaciones en forma de texto'. La visualización tipo texto proporciona una representación de la realidad para ser 'leída': gráficos, tablas, mapas, etc." (Verbeek, 2001: 140-142).

El resultado de estos enfoques es estudiar el "papel que desempeñan las tecnologías concretas en nuestra cultura y en la vida cotidiana de las personas" (Verbeek, 2001: 120) porque "las cosas son lo que son en virtud de nuestras relaciones con ellas", y "la tecnología es dominación y control" (Verbeek, 2001: 121). 
Incluso si la tecnología tiene efectos en nuestras vidas, esto no significa que seamos conscientes de ellos. "Las tecnologías dan lugar a un «campo» de fondo en el que podemos tener experiencias sin experimentar explícitamente estas tecnologías.Están presentes y ausentes al mismo tiempo: sin que nos demos cuenta, ellas dan forma a nuestra experiencia configurando un contexto" (Verbeek, 2001: 136).

"Las tecnologías proporcionan un marco para las acciones humanas; tienen una cierta influencia en esas acciones. Esta influencia no es una determinación, sino una inclinación. Las tecnologías 'quieren' que las personas hagan las cosas de manera particular, por así decirlo: tienen cierta 'intención' y promueven esta intención entre sus usuarios" (Verbeek, 2001: 136). Esta es la razón por la cual es necesario estudiar estas influencias. Si las implicaciones tecnológicas se nos presentaran claramente, no sería necesario pensar en ellas. Así, como Durkheim menciona, "la vida social debe explicarse, no por la concepción que de ella tienen los que en ella participan, sino por causas profundas que escapan a la conciencia" (Durkheim, 1982:30). En consecuencia, debemos estudiar qué mundos son posibles debido a estos artefactos, y cómo estos objetos cambian nuestra percepción sobre el mundo.

Consideremos el caso de la comunidad médica. De acuerdo con la familiaridad que desarrollan en sus estudios con el entorno médico, creen que es natural como son las cosas. No obstante, su práctica es producto de un entorno cultural lleno de desarrollos tecnológicos, unos en forma de objetos, y otros en forma de prácticas. Usan herramientas para mejorar sus sentidos, como: estetoscopios, otoscopios, rinoscopios, brazaletes de presión arterial, entre otros; los cuales les permiten amplificar o reducir sus sentidos. Los médicos están organizados de una manera particular, para mejorar su desempeño a través de la burocracia, la división social del trabajo por especialidades y la administración. El conocimiento médico proporciona los medios para leer la información que circula en la práctica. También la práctica médica es posible gracias al establecimiento de ciertos espacios, que son igualmente el resultado del conocimiento aplicado, como el hospital (Foucault, 1985).

La relación de todas estas tecnologías hace posible un espacio construido culturalmente, donde se encuentran las acciones y experiencias de la práctica médica. Se convierte en una relación entre la percepción sensorial de los científicos, y el contexto, en palabras de Ihde: "la ciencia no solo debe relacionarse con el contexto de interpretación en el que se lleva a cabo, sino también con las percepciones sensoriales de los científicos" (Ihde en Verbeek, 2001: 140). 


\section{¿Qué es el lenguaje en la práctica médica? Dr House como ejemplo}

La serie Doctor House, emitida en el año 2004, gira en torno al médico Gregory House, quien se encarga de resolver casos médicos complicados que otros doctores no pueden resolver. House cuenta en su equipo con los médicos Eric Foreman, Allison Cameron y Robert Chase, quienes forman parte del Departamento de Diagnóstico Médico del Hospital Princeton Plainsboro. House se caracteriza por ser un médico que, a diferencia del resto, no se preocupa por salvar vidas, sino que ve a los enfermos como enigmas que hay que resolver. Esto quiere decir, que si un paciente suyo muere, éste no lo reconoce como "caso cerrado", sino al contrario, continúa haciendo exámenes y relacionando los síntomas, con el fin de obtener un diagnóstico y, así conocer la enfermedad que provocó su muerte.

Esta serie pone en evidencia cómo, para House, el funcionamiento del cuerpo humano sigue la lógica de un sistema, en el que al fallar uno de sus componentes, se presenta un síntoma médico; por lo tanto, es necesario encontrar el punto donde el sistema falla, como si de una máquina se tratara, para encontrar la enfermedad que lo causó. De esta manera, para House, la enfermedad se constituye como un enigma a resolver. En consecuencia, nos preguntamos, cómo un médico puede entender a un paciente como un enigma.

Vemos en la serie cómo House escribe en una pizarra los síntomas que tienen los pacientes $\mathrm{y}$, a partir de la relación de los síntomas que va encontrando, trata de establecer un diagnóstico, o sea, encontrar la enfermedad que se corresponde con esos síntomas. La noción, que tiene House, del sistema-cuerpo es abstracta, por eso basta con hacer un inventario de síntomas para saber de cuál enfermedad se trata. No es necesario conocer el contexto particular ${ }^{2}$ de cada paciente, su forma de ser y vivir, sino que es suficiente con observar los síntomas que el cuerpo manifiesta, y que se codifican gracias a la labor de otros médicos que llenan extensos formatos para que el médico encargado de realizar el análisis dé su concepto.

House es el paradigma ${ }^{3}$ de un ideal médico moderno que, a diferencia de la manera en que se ejercía la medicina anteriormente, puede omitir el contacto físico con el paciente, ya que no cree necesario saber las condiciones en que estos se encontraban al momento de generarse un síntoma. Es suficiente concentrarse en los síntomas, y leerlos como información, como datos que dan cuenta de una enfermedad.

\footnotetext{
1 House le hace muchos guiños a Sherlock Holmes, figura literaria detectivesca que a su vez estuvo basada en un doctor escocés.

2 En este punto entendemos por contexto las características personales del paciente, como su historia de vida en general, sus apreciaciones emocionales o la relación médico paciente; ya que House a veces revisa las casas de los usuarios como parte de su investigación. Pero ello es parte del modelo empiricista de buscar pistas para descubrir la verdad, modelo que deja de lado muchos aspectos importantes.

3 Un paradigma es un "caso ejemplar que exagera las características de las situaciones del mismo genero para mostrar que tienen en comun todos los casos". En este sentido, "el paradigma es una forma de conocimiento no inductiva, sino analógica que se mueve de la singularidad a la singularidad. (...) El caso paradigmático deviene tal suspendiendo y, a la vez, exponiendo su pertenencia al conjunto, de modo que ya no es posible separar en él ejemplaridad y singularidad" (Agamben, 2011: 11).
} 
A pesar de que el médico se relacione cuerpo a cuerpo con su paciente, y haga un proceso de observación sin la intervención de máquinas o suplementos que le permitan ver los síntomas, el proceso de diagnóstico es siempre un proceso lingüístico, ya que el médico observa los síntomas en el paciente como signos de la enfermedad (Foucault, 1985). Para Saussure (2005), un signo se compone de un significado y un significante, donde el significado es un concepto o una idea, y el significante es una imagen acústica; en este caso, una partición sensorial. Así, el procedimiento que se establece a la hora de identificar un síntoma es asociar algo que el médico ve, una magulladura, sangre, hemorragias, pus, irritaciones, etc., con un concepto-idea médica.

Lo anterior significa que cuando un médico observa una magulladura en el cuerpo de un paciente, relaciona ese signo con el resto de signos que componen un lenguaje. Una enfermedad sólo puede ser diagnosticada cuando el médico identifica los signos que la constituyen, construyendo así una semiotica médica. Como consecuencia, el cuerpo del paciente solo puede ser aprehendido por el médico gracias a una conversión de ese cuerpo a un lenguaje clínico, que pasa por un procedimiento establecido de: lectura-observación de síntomas, sistematización y análisis de resultados, registro de formularios y, finalmente, medicación para tratar la enfermedad. Este procedimiento se constituye en lo que Michel Foucault llama "Tecnología del poder"; es decir, como una concatenación de acciones que tienen como fin dirigir la conducta (Castro, $2011)^{4}$.

Los médicos tienen unas prótesis físicas que les permiten percibir el cuerpo de los pacientes de formas diferentes a la manera que lo harían sólo con sus sentidos. Merlau-Ponty nos ayuda a comprender que "los seres humanos no solo pueden extender la espacialidad de sus cuerpos vividos con la ayuda de artefactos, sino también percibir con ellos" (Merleau-Ponty, 1962:143). Los médicos convierten la percepción que obtienen, con la ayuda de estos objetos, en un lenguaje que consiste en sonidos (con ayuda del estetoscopio), imágenes, y gráficos (a través de radiografías y otros similares) que le permite al médico comprender lo que le está sucediendo al paciente. En otras palabras, los médicos hacen que el paciente y sus enfermedades sean aprehensibles.

Estas máquinas codifican el cuerpo de sus pacientes y dejan como resultado datos sobre el cuerpo. Los médicos, entonces, no tratan directamente con el cuerpo, sino con los datos que se crean a partir de él. Lo que el médico interpreta no es el cuerpo humano, sino la organización de los datos que ha recopilado. Estas conversiones que el lenguaje hace del cuerpo se basan en una interrelación de percepciónes, nociones, prácticas, discursos, máquinas y dispositivos. A partir de lo anterior, podemos afirmar que los médicos no tratan directamente con el cuerpo, en tanto que su relación siempre esta mediada por un conjunto de tecnologías. Aun cuando el cuerpo del médico está en contacto físico con el cuerpo del paciente, su relación está medidada por un conjunto de saberes y un contexto especifico que posibilita esa relación.

4 Es importante resaltar que el poder no se reduce a una imagen de control y sumisión, sino que el poder tiene facultades creativas y de resistencia. Foucault explica que: "el poder no debe ser entendido como un sistema opresivo que somete desde la altura a los individuos, castigándolos con prohibiciones sobre esto o aquello. El poder es un conjunto de relaciones. ¿Qué significa ejercer el poder? (...) Ejerzo poder sobre ti: influyo en tu comportamiento o intento hacerlo. Intento guiarlo, conducirlo" (Foucault, 1988). 
Por lo tanto, en la práctica médica no sólo existe "el lenguaje medico", sino que existen diferentes elementos que construyen un dispositivo.

Para entender por qué la práctica médica funciona como un dispositivo, es fundamental recordar la definición de Giorgio Agamben. Según Agamben, un dispositivo es "todo aquello que tiene, de una manera u otra, la capacidad de capturar, orientar, determinar, interceptar, modelar, controlar y asegurar los gestos, las conductas, las opiniones y los discursos de los seres vivos" (Agamben, 2011). En este sentido, se debe observar cómo se organizan, y qué hacen los sujetos, las nociones que tienen del mundo, la manera cómo utilizan las máquinas, cómo las máquinas enfocan la percepción, y qué discursos se manejan en un campo determinado. En este caso, nos referimos a la práctica médica.

La práctica médica funciona como un dispositivo que se encarga de capturar, orientar, determinar, interceptar, modelar, controlar, y asegurar, los gestos, las conductas, las opiniones y los discursos de los seres vivos, en la medida que crea un sistema que contiene: sujetos, los doctores; objetos, los instrumentos tecnológicos que orientan la observación del médico; códigos, como resultado de la conversión del cuerpo en datos; y normatividades, pues los médicos actúan a partir de unos parámetros legales ${ }^{5}$, tales como el consentimiento informado, por ejemplo, en el caso colombiano, la Ley $100^{6}$, entre otros, que permiten apaciguar, o dejar de lado, la cuestión moral.

Ahora bien, hemos denominado a las situaciones seleccionadas de cada capítulo como: "Comité de trasplante", "Tecnología de punta" y "Consentimiento informado". Tomamos estos fragmentos porque nos posibilitan observar algunos de los diferentes componentes mencionados, y la forma cómo se relacionan en el dispositivo médico. A continuación, se realizará el análisis de los fragmentos seleccionados.

\section{"Comité de trasplante" fragmento del capítulo 14 de la primera temporada:}

Una mujer necesita un trasplante de corazón, por lo que su nombre es puesto en la lista de espera. Para que el caso de la mujer pueda ser priorizado, es necesario que cumpla con una serie de requisitos; por ejemplo, que no tenga una enfermedad terminal, que no abuse de sustancias alcohólicas o psicoactivas, que no presente una condición psiquiátrica, entre otros aspectos. Los miembros del comité de trasplante son los encargados de evaluar el caso de la mujer, en sus manos está que se le permita el acceso al órgano que requiere, no sólo de acuerdo a su disponibilidad, sino también, conforme al cumplimiento de las condiciones establecidas.

\footnotetext{
5 En el caso colombiano podemos encontrar normatividades como: el Triaje, tarifas SOAT, Manual de medicamentos del POS y Guías para manejo de urgencias. Estos documentos estandarizan la relación médico-paciente al estar atravesada por un proceso burocrático por medio del cual se establece cómo tratar a los pacientes y sus enfermedades.

6 La ley 100 de 1993, decretada durante el gobierno del presidente Cesar Gaviria (1990-1994), fue propuesta por, el también ex presidente de la república, Alvaro Uribe Velez. Esta ley se caracteriza por el impulso a organizaciones privadas externas por el control del sistema de salud a nivel nacional lo cual ha generado una restricción en el acceso a los servicios de salud por parte de las entidades aseguradoras frente a las poblaciones de bajos recursos económicos. Así, la ley 100 de 1993 se caracteriza por ser una normatividad que vuelve al cuerpo mercancía, en la medida en que el acceso al sistema de salud colombiano depende más de la capacidad adquisitiva (en términos económicos) de los usuarios del sistema, que de la urgencia de los tratamientos de los pacientes.
} 


\section{Transcripción del diálogo:}

Cuddy: “¿Hay algún criterio de exclusión del que debamos saber?”

House: "La tomografía no revelo tumores y el doctor Wilson no encontró rastros de cáncer".

Cuddy: "Dígame, ¿hay otro criterio?”

House: "No hay enfermedad arterioesclerótica vascular, no hay neumonía ni bacteriemia, o herpes B o C o ninguna otra letra".

Cuddy: "¿Abusa de sustancias, alcohol, drogas? ¿alguna condición psiquiátrica, historial de depresión? Dr. House si se insubordina o engaña a este comité va a ser sujeto a una acción disciplinaria ¿Hay algún criterio de exclusión en el receptor que descalificaría a su paciente para conseguir un corazón?”

House: "No".

En este caso, se evidencia cómo la práctica médica se puede comprender, en términos de Agamben, como el ejercicio de un poder soberano que consiste en hacer morir y dejar vivir ${ }^{7}$. El médico cumple el papel del soberano, y el paciente de súbdito; la vida de éste último depende de las decisiones que tome el soberano con respecto a una situación en particular. De este modo, un trasplante de órganos depende más de unos criterios médicos que determinan si el paciente es apto, o no, para recibir un órgano, que de la disponibilidad de éste. Entonces, si un paciente cumple con los criterios establecidos por el comité, tendrá mayor posibilidad de que su posición en la lista de espera ascienda, y su caso merezca ser priorizado para garantizar la obtención del órgano que necesita. Se ve entonces que un elemento central del dispositivo medico es el poder que ejercen los médicos.

\section{"Tecnología de punta" fragmento del capítulo 10 de la segunda temporada:}

Los miembros del equipo médico de House, Cameron y Foreman, han realizado varias pruebas con diferentes instumentos tecnológicos, para averiguar los efectos de la cirugía practicada a un paciente que sufría de desórdenes psicológicos. No obstante, luego de analizar las muestras a través de diferentes aparatos médico-tecnológicos, como no encontraban las causas de los síntomas que presentaba el paciente, deciden hacer una pueba ellos mismos. Los médicos colocan la sangre del paciente en una platina, y la observan por el microscopio; en ese momento, descubren qué tiene el paciente, y proceden a dar un diagnóstico.

\footnotetext{
7 "El soberano tiene la capacidad y la legitimidad de ejercer un tipo de poder sobre la vida de los súbditos por lo que si lo dispone puede arrebatárselas. El poder soberano es, en sí mismo, un estado de excepción en el que el soberano se encuentra tanto fuera como dentro de la ley. Según Agamben "el soberano, por medio del estado de excepción, <<crea y garantiza la situación $>>$ de la que el derecho tiene necesidad para su propia vigencia” (Agamben, 2010: 29).
} 


\section{Transcripción del diálogo:}

Foreman: "Si un ser humano hubiera visto esta sangre en alguna parte del proceso, en lugar de hacer pruebas con computadoras, los parásitos le habrían saltado encima”.

Cameron: "El precio de la era electrónica”.

En este caso, los médicos siguen una serie de procedimientos para poder establecer un diagnóstico frente a lo que el paciente presenta. Sin embargo, se ha creado una barrera entre el médico y el paciente, ésta ha causado que el médico se abstenga de examinar al paciente, de tener contacto físico con él. Por ello, el médico recurre a instrumentos tecnológicos que analizan, en este caso, la sangre del paciente, y la labor del médico es realizar una lectura de los resultados que la máquina le muestra. Así pues, no es el cuerpo del paciente el que es examinado por el médico, sino que es analizado a través de una herramienta médica; entonces, se realiza una lectura de cifras, porcentajes e índices como resultado de las pruebas realizadas.

\section{"Consentimiento informado" fragmento del capítulo 3 de la tercera temporada}

Una interesante conversación se genera entre la doctora Cameron y un investigador de cáncer que se encuentra hospitalizado debido a problemas respiratorios. En esta conversación, Cameron lo acusa de haber irradiado bebés humanos en medio de una experimentación para encontrar la cura del cáncer; frente a esta imputación el hombre se defiende asegurando la efectividad del consentimiento informado. Él asegura no sentirse arrepentido ya que había salvado muchas vidas al encontrar la cura de esta enfermedad.

\section{Transcripción del diálogo:}

Cameron: "House quiere una biopsia de su piel y me envió a conseguirla" Hombre: "Ahh y tú estás de acuerdo"

Cameron: "No tuve nada que ver con ponerlo en coma o los exámenes posteriores"

Hombre: "Pero tú estás aquî"

Cameron: "Leí algunos de sus artículos"

Hombre: "Hubo muchos de ellos"

Cameron: "Revista médica de Massachusetts del 67' irradió bebés, tan sencillo, sin formulario, ni consultas, nada. ¿Cuántos enfermos de cáncer causó?” 
Hombre: "No lo sé. Lo que sé es que descubrí técnicas que previeron fallas renales mortales en miles de otros niños"

Cameron: "¿Y lo dice así?”

Hombre: "No me arrepiento de lo que hice. Consentimiento informado, derechos del paciente demorados para investigación" [Cameron toma el brazo derecho del paciente y le corta un pedazo de piel] “¿Qué hiciste?”

\section{Cameron: "El consentimiento informado demora nuestro diagnóstico"}

Hombre: "Bien por ti, ya sales en defensa de algo actuando por lo que crees”.

El consentimiento informado es un documento que autoriza al médico una intervención de determinado tipo sobre el paciente; esta intervención puede ser experimental, o con fines investigativos, por lo cuál puede poner en riesgo la vida del paciente, ya que no se tiene certeza de los resultados e implicaciones del procedimiento. En este sentido, las consecuencias de un determinado procedimiento recaen en el paciente, y no son responsabilidad del médico, puesto que este documento sirve como un soporte que indica la aceptación de los riesgos por el paciente.

Como se puede observar, ésta es otra forma de separar el vínculo entre paciente y médico y así descargar el conflicto moral ${ }^{8}$ en un proceso burocrático, es decir, lo que media la relación es un documento.

Teniendo en cuenta estas tres situaciones, se debe resaltar cómo la noción de dispositivo visibiliza cierto tipo de relaciones en la práctica médica, que dan cuenta de la conversión de la vida en datos, es decir, de la conversión del cuerpo a un lenguaje médico especifico. Las tres situaciones ponen en evidencia cómo la toma de decisiones, por parte de los médicos, se da a partir de unos criterios de selección que inciden directamente en la vida ${ }^{9}$ de las personas; dígase, condiciones para recibir o no un órgano, la lectura de datos generados por un instrumento tecnológico, y la firma de un documento que libera al médico de toda responsabilidad sobre el paciente. Dr. House es un caso paradigmático para estudiar la práctica médica, a través de modelos, porque nos permite resaltar la importancia de la conversión del cuerpo en datos como elemento escencial del ideal médico contemporáneo.

A continuación, para comprender cómo funciona esta codificación del cuerpo en datos, veamos un ejemplo emblemático ${ }^{10}$ de un paciente con una dolencia en el corazón que es atentido en el sistema de salud colombiano. Lo anterior con la intención de mostrar la continuidad entre los hallazgos encontrados en el análisis de la serie, y las condiciones reales del ejercicio médico.

8 La cuestión de la moral en la práctica médica fue tratada en la ponencia "Economía moral y bioética: Hacia un método de análisis antropológico de situaciones clínicas”, presentada por Clavijo y Ospina (2015).

9 Todo se lee ahora a partir de códigos. Se codifican los cuerpos para así descifrarlos, leerlos como datos. Se trata de un proceso mecánico en el que el médico se encarga de comprender los signos del cuerpo, a partir de cifras y porcentajes para así generar un diagnóstico.

10 Este es un caso real. El paciente fue entrevistado por los autores de este artículo con el fin de conocer su experiencia en el sistema de salud colombiano. El entrevistado será mantenido en el anonimato y será nombrado como "paciente X". 
El paciente X recurrió al servicio de urgencias por un dolor punzante en el corazón, al llegar fue clasificado su nivel de urgencia a través de un "triaje"11, realizado por una enfermera. Luego de que la enfermera ha determinado la clasificación a la que corresponde la dolencia del paciente, aunque sin darle a él una explicación sobre ésta, le pide que se dirija a la sala de espera. Media hora después, llamaron al paciente X para realizarle un electrocardiograma y, en seguida, lo mandaron de nuevo a la sala de espera diciéndole:

"salga y espere a que lo llamen". Pasada otra media hora, lo atendió un médico; éste lo recibe con el electrocardiograma en la mano.

Todas las urgencias cardiacas tienen un protocolo que consiste en que se haga una revisión por parte de un médico general, y las conclusiones las mira de nuevo el médico jefe de la unidad. Por este motivo, se le realizó un electrocardiograma antes de que el médico chequeara al paciente, para que así, con los resultados del examen, éste tuviera la información necesaria para generar un diagnóstico, y remitir al paciente a la especialidad pertinente.

La persona que realiza el electrocardiograma, y el médico que atiende al paciente, son personas diferentes y están mediadas por un proceso burocrático, asignado por el hospital. El trato, en general, con el médico, fue muy corto ya que el médico se limitó a leer el electrocardiograma y, en seguida, manifestó: "usted tiene una posible arritmia, a usted toca remitirlo al cardiólogo". Inmediatamente le explican la lectura del electrocardiograma al paciente, y le recetan aspirinas; también le piden hacerse un holter y un ecocardiograma, previos a la cita con el cardiólogo.

En la cita con el cardiólogo, éste le explica la importancia de descartar alguna anomalía fisiológica, antes que nada; el médico llegó a esta conclusión al ver el ecocardiograma. El médico no lee todos los resultados de la prueba del holter, sino que revisa el resumen que ha elaborado anteriormente el otro cardiólogo encargado de realizar este examen. El entrevistado argumenta que pareciera que los médicos se apoyan en los hombros del otro. Posteriormente, el cardiólogo envió al paciente $\mathrm{X}$ al electrofisiólogo.

Un médico, amigo del paciente $\mathrm{X}$, haciendo mofa de la situación por la que estaba atravesando X, afirmó: "hoy en día un cardiólogo es como un médico general"12. Cuando el paciente X llega al electrofisiólogo, quien lo atiende es un médico que vuelve a leer los exámenes, haciendo un resumen y una interpretación del caso para el electrofisiólogo. El especialista no habla con el paciente, sino con el médico que hizo el resumen, mientras ojea los exámenes. En este caso, el especialista afirmó estar de acuerdo con el diagnóstico hecho por el médico, así como con la medicación y el tiempo de medicación.

11 El Triaje es un método de selección y clasificación de pacientes empleado en la medicina de emergencias y desastres. Evalúa las prioridades de atención, privilegiando la posibilidad de supervivencia, de acuerdo a las necesidades terapéuticas y los recursos disponibles. En situaciones de demanda masiva, atención de múltiples víctimas o desastre, se privilegia a la víctima con mayores posibilidades de supervivencia según gravedad y la disponibilidad de recurso. En Colombia el triage debe ser realizado a la llegada del paciente al servicio de urgencias.

12 Esta frase se sustenta en que hoy en día quien diagnostica y resuelve los problemas cardiacos no es el cardiólogo, sino el especialista que corresponda a la zona del daño, de esta manera, el cardiólogo, aún siendo un especialista se vuelve sólo un intermediario, como un médico general. 
Se debe resaltar que el electrofisiólogo pasa recorriendo varios consultorios por un área específica del hospital, el paciente X, por tanto, no estaba en su consultorio. El tiempo que el paciente $\mathrm{X}$ estuvo en presencia del electrofisiólogo fue entre cinco y diez minutos. La relación del médico y el especialista es de completa sumisión, llegando al punto de llamarlo, a él, "doctor". Posterior a esto, el médico continúa llenando los formatos; según la interpretación del paciente $\mathrm{X}$, la función del médico ahí es optimizar la intervención del electrofisiólogo, encargándose de todo el papeleo. En esta consulta, al paciente $\mathrm{X}$ lo examinaron tomándole la presión, midiéndolo, y pesándolo.

A partir de la noción agambeniana de dispositivo, podemos ver el conjunto de relaciones que se entrelaza para posibilitar el tratamiento del paciente en cuestión. Por un lado, la división del trabajo entre administradores, burocráticos, enfermeras y doctores, incluso la estratificación entre los mismos médicos. Por otro lado, el conjunto de normatividades como el triaje, atención de urgencias, etc., que destinan espacios y tiempos para la atención y, además, estandarizan los distintos procedimientos a seguir con el paciente. La conversión del cuerpo del paciente en datos, emerge de la relación entre las normatividades, los médicos, especialistas, enfermeras, el sistema burocrático y el paciente.

El paciente experimentaba la sensación de dolor sin la capacidad de señalar con precisión en qué parte del cuerpo lo sentía. Podía referirse a la zona, o al área, en la que sientía dolor, pero no tenía la habilidad para nombrar, desde el lenguaje médico, su condición. Algo tan indeterminado, como lo es el dolor, empieza a ser focalizado, aislado y reglamentado a partir del conjunto de preguntas estandarizadas, el uso de las tecnologías, y la aplicación de un saber. Estos procedimientos permiten "desligar el dolor" del paciente y, de esta forma, convertir la dolencia en un conjunto de datos que pueden ser leídos por otro especialista que, para diagnosticar, tiene que utilizar las tablas de diagnóstico que proponen ciertas normatividades para dolencias específicas, dejando al cuerpo como un elemento secundario en el proceso de curación.

Luego del análisis del caso del paciente $\mathrm{X}$, atendido por el sistema de salud colombiano, y al comprender la serie de Dr. House como un caso paradigmático de la práctica médica colombiana, consideramos pertinente cuestionarnos de qué manera incide la codificación del cuerpo en la constitución de las distintas normatividades, cómo se configura la relación médico-paciente, qué nociones del cuerpo permite, cómo posibilita la división del trabajo médico, y los distintos procesos de diagnósticos.

Se propone aplicar estas preguntas a diferentes casos en América Latina como una agenda investigativa que nos permita relacionar los distintos elementos que componen la práctica médica, entre ellos, las normatividades, los procedimientos estandarizados, la relación local médico-paciente, la conversión del paciente en datos, y las implicaciones de la codificación del cuerpo.

La burocratización de la medicina sólo es posible gracias a esta división de la información; las distintas tecnologías aseguran la codificación del cuerpo y, de esta manera, la mercantilización de la salud ${ }^{13}$.

13 Para profundizar en la relación codificaciones del cuerpo, significados de la vida y mercantilización de la salud puede referirse al 
Sería importante reflexionar sobre este último punto para entender como una totalidad a la práctica médica, y no como puntos aislados sin interconexion.

A pesar de que existan procesos globales de construcción conceptual del cuerpo, y establecimiento de tecnologías que guían las percepciones, el encuentro médico no fluye en una sola dirección. Las personas tienen un margen de interpretación y elección individual. La práctica médica no es una realidad monolítica; los niveles de autoritarismo y autonomía personal varían de acuerdo al contexto específico. En este artículo, abordamos una versión idealizada del nuevo paradigma que atraviesa la práctica médica contemporánea, no obstante, los sistemas de salud del mundo se caracterizan también por la ausencia y precariedad y, en ese sentido, hay muchos contextos en donde la práctica médica es muy frágil y no tiene mucha capacidad de control.

Finalmente, podemos ver que, incluso las cosas más cercanas que afectan nuestras vidas, como lo son los procedimientos que los médicos usan para curar, están atravesadas por la tecnología y la ciencia. Elegimos este ejemplo para demostrar que, no sólo las tecnologías más sorprendentes se pueden estudiar desde estas perspectivas, incluso los dispositivos más desapercibidos son importantes para codificar y decodificar el cuerpo y, así, revelar nuevos mundos. Las percepciones que tienen los expertos están mediadas por los antecedentes tecnológicos y los dispositivos que tienen en su profesión. En consecuencia, su percepción no es natural, sino que es una construcción social que tiene implicaciones directas en la forma cómo se relacionan con otros seres sociales. Además, estas percepciones, como señala Ihde, están influenciadas, o dirigidas, por la configuración interna que tienen estos objetos. Como conclusión, para entender al ser humano y las relaciones sociales en las que está involucrado, debemos tener en cuenta los aportes que nos brinda la filosofía de la ciencia y la tecnología.

capitulo de libro, de Jairo Clavijo, "Humanización de la salud, cosificación y significados de la vida y de lo viviente en el sistema de salud colombiano" en el libro La humanización de la salud conceptos, criticas y perspectivas (2017). 


\section{Bibliografía}

Agamben, Giorgio. (2008). ¿Qué es un paradigma? En: Revista FRACTAL, revista iberoamericana de ensayo y literatura. No. 48. Recuperado de: https://mxfractal.org/ articulos/RevistaFractal53GiorgoAgamben.php

TEXTOS.

(2010). Homo sacer I. El poder soberano y la nuda vida. España: PRE-

------- (2011). ¿Qué es un dispositivo? En: Sociológica, No. 73, pp. 249-264. México.

Castro, Edgardo. (2011). Diccionario de Foucault. Buenos Aires, Argentina: Siglo $\mathrm{XX}$.

Clavijo, Jairo y Ospina, Juan Camilo. (2016). Economía moral y bioética: Hacia un método de análisis antropológico de situaciones clínicas [Ponencia]. En: XII Congreso Internacional Sociedad Hispánica de Antropología Filosófica. España: Universidad de Zaragoza.

Clavijo, Jairo. (2017). Humanización de la salud, cosificación y significados de la vida y de lo viviente en el sistema de salud colombiano. En: La humanización de la salud conceptos, criticas y perspectivas. Bogotá: Pontificia Universidad Javeriana.

Durkheim, Emile. (1982). Las reglas del método sociológico. Barcelona: Editores Orbis

Fassin, Didier. (2004). Entre las políticas de lo viviente y las políticas de la vida. Hacia una antropologia de la salud. En: RCA, 40, 283-318.

Foucault, Michel. (1985). El Nacimiento de la clínica. Mexico: Siglo XXI.

(1988). Entrevista a Michel Foucault: El poder, los valores morales y el intelectual. Recuperado de: https://www.michaelbess.org/foucault-interview/ (1994). "Dits et Ecrits Tome IV" Paris, Francia, Broche.

Good, Byron. (1994). Medicine, Rationality and Experience: An Anthropological Perspective. Cambridge: Cambridge University Press

Heidegger, Martin. (1996). Being and Time. USA: State University of New York Press.

Merleau-Ponty, Maurice. (1962). Phenomenology of Perception. London: Routledge.

Normatividades: Ley 100 de 1993; Manual de tarifas SOAT, Guía para Manejo de Urgencias, Manual de medicamentos del POS y Triaje.

Pertierra, Anna Cristina. (2018). Media Anthropology for the Digital Age. UK: Polity Press.

Saussure, Ferdinand. (2005). Curso de lingüística general. Bogotá, Colombia: Editorial AKAL.

Verbeek, Peter-Paul (2001) «Don Ihde: The technological lifeworld» in «American Philosophy of technology. The empirical truth». USA: Indiana University Press. 